\title{
Carbon dots and CdTe QDs hybrid-based fluorometric probe for spermine detection
}

Yanzhao Fư ${ }^{\dagger \neq, 1}$, Shuilin $\mathrm{Wu}^{\S, 1}$, Hongkang Zhou ${ }^{\square, 1}$, Shaojing Zhao ${ }^{\dagger}$, Minhuan Lan ${ }^{\dagger}, *$, Jufang Huang ${ }^{\square, *}$, and Xiangzhi Song ${ }^{\dagger}$ **

${ }^{\dagger}$ Key Laboratory of Hunan Province for Water Environment and Agriculture Product Safety, College of Chemistry and Chemical Engineering, Central South University, Changsha, 410083, P. R. China.

* Shenzhen Research Institute of Central South University, Shenzhen, 518057, P. R. China.

$\S$ Center Of Super-Diamond and Advanced Films (COSDAF) \& Department of Materials Science and Engineering, City University of Hong Kong, Hong Kong SAR, P.R. China.

$\square$ Department of Anatomy and Neurobiology, School of Basic Medical Sciences, Central South University, Changsha, 410013, P.R. China.

${ }^{1}$ These authors contributed equally to this work.

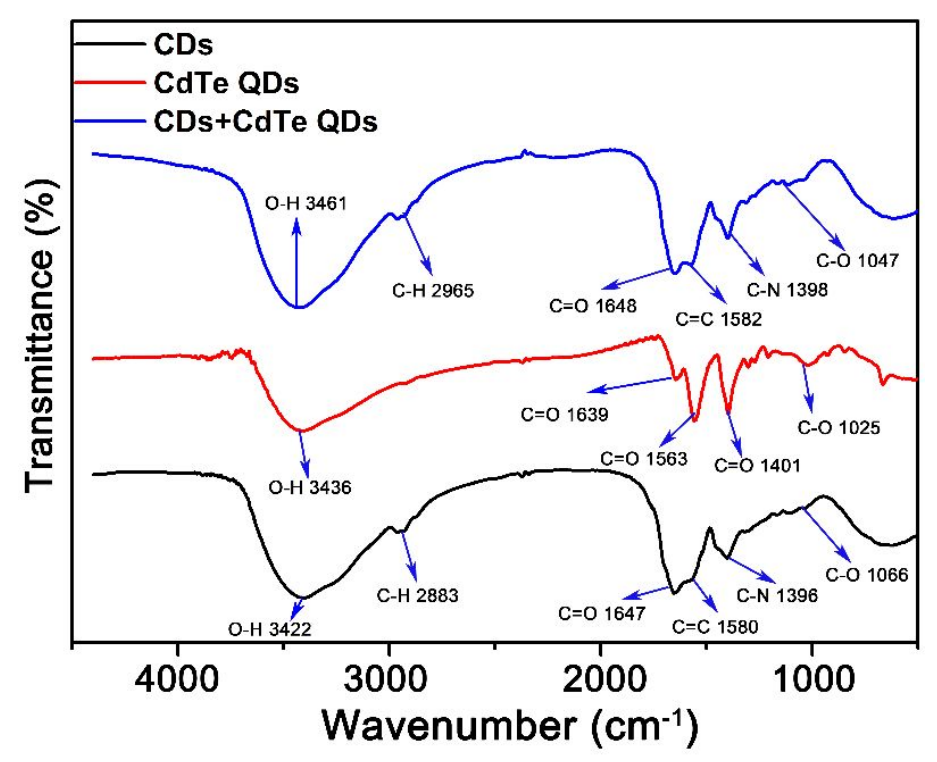

Figure S1. FT-IR spectra of CDs (black line), CdTe QDs (red line), and CDs+CdTe QDs hybrid (blue line). 

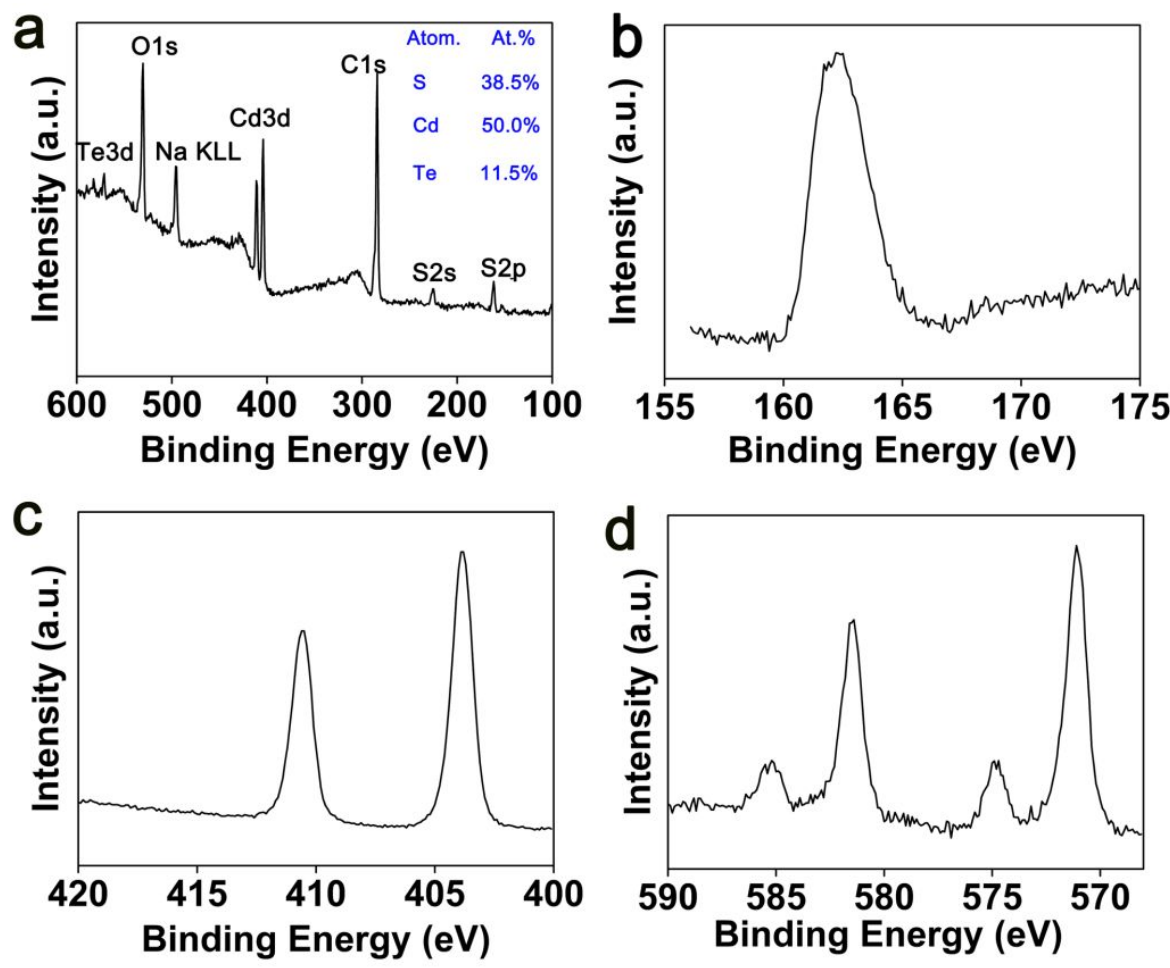

Figure S2. (a) XPS survey spectrum of CdTe QDs. High-resolution XPS spectra of (b) S2p, (c) Cd3d and (d) Te3d.
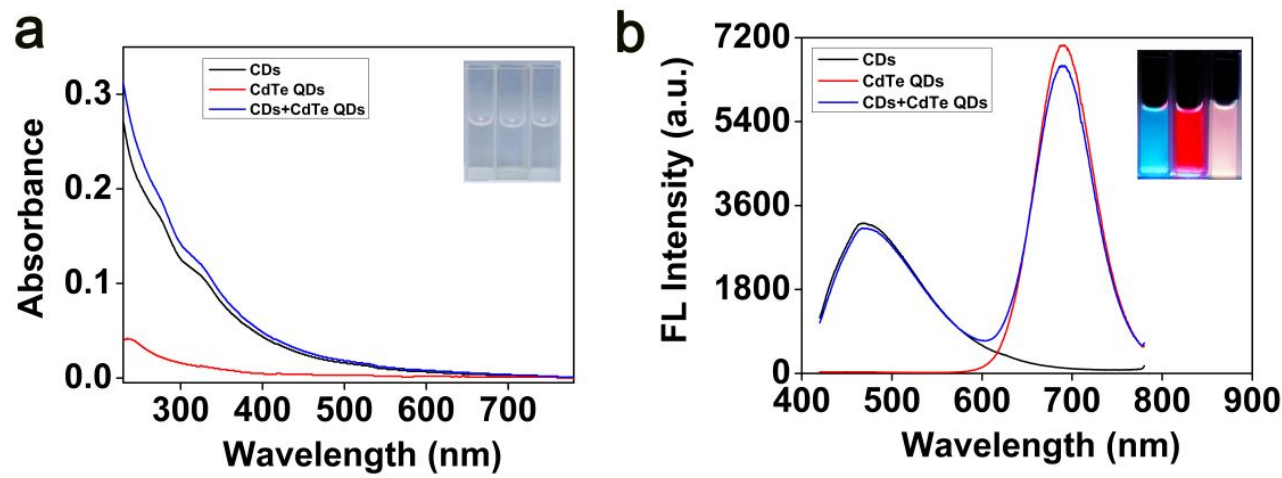

Figure S3. (a) Absorption and (b) fluorescence spectra of the CDs, CdTe QDs, and CDs+CdTe QDs hybrid solutions. Insets: photographs of CDs, CdTe QDs, and CDs + CdTe QDs hybrid solution (from left to right) under the (a) white light and (b) $365 \mathrm{~nm}$ UV light irradiation, respectively. 

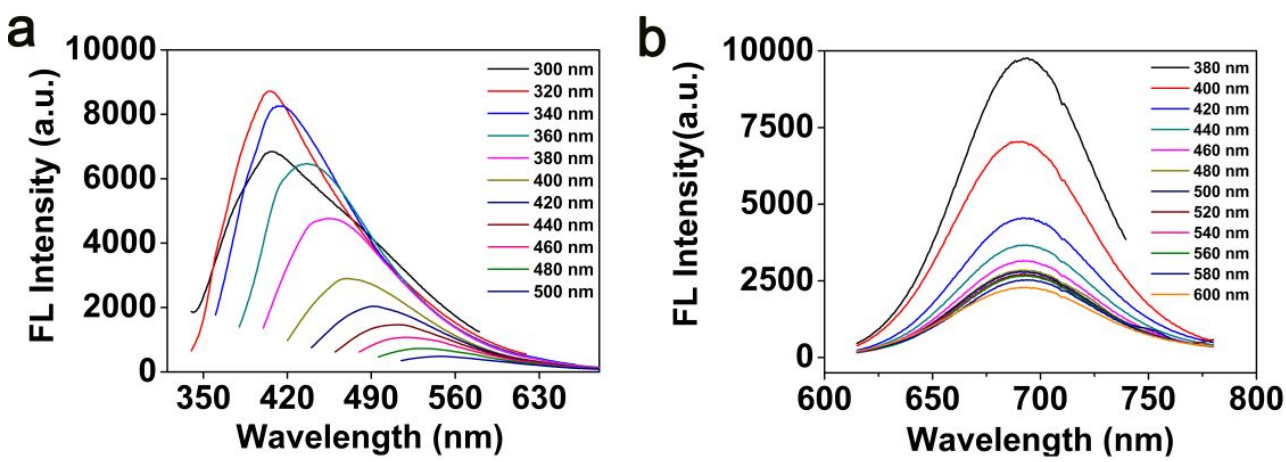

Figure S4. Fluorescence spectra of (a) CDs and (b) CdTe QDs aqueous solutions under different excitation wavelengths.
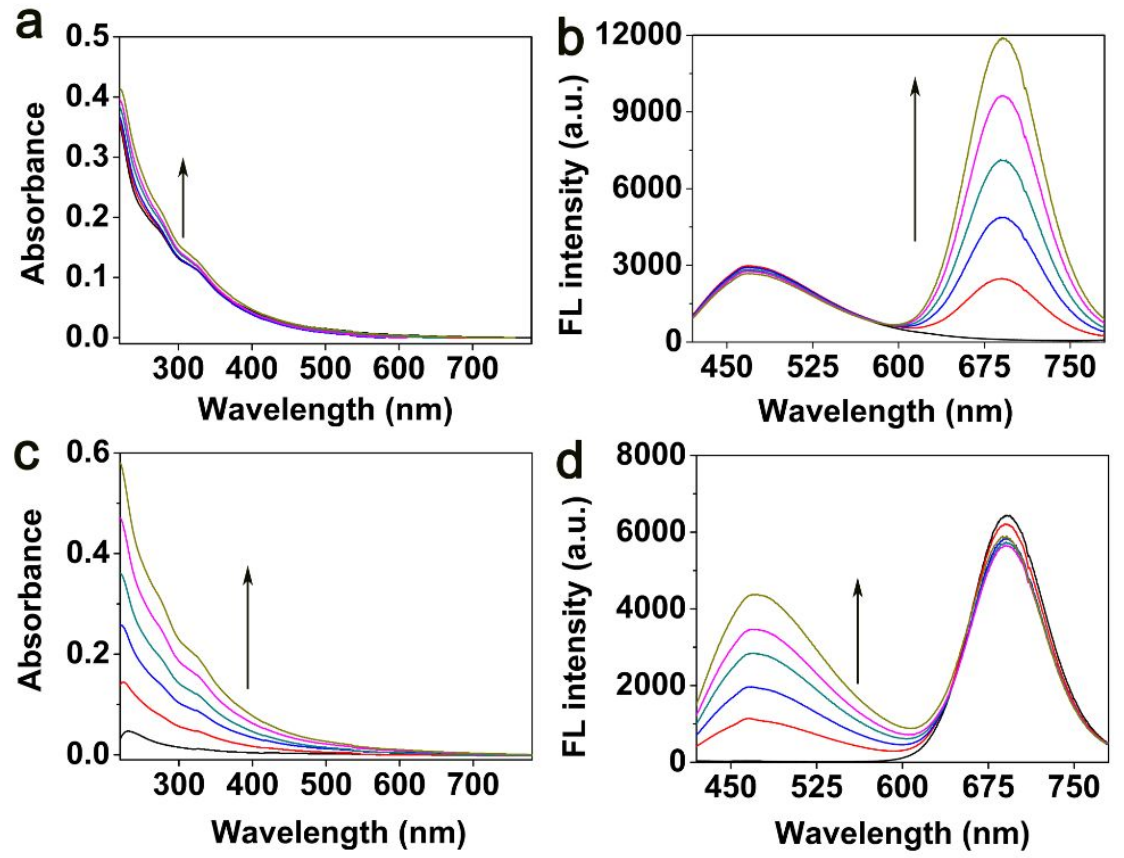

Figure S5. (a) Absorption and (b) fluorescence spectra of CDs aqueous solution with the gradual addition of CdTe QDs from 0 to $60 \mu \mathrm{g} / \mathrm{mL}$. (c) Absorption and (d) fluorescence spectra of CdTe QDs aqueous solution with the gradual addition of CDs from 0 to $750 \mu \mathrm{g} / \mathrm{mL}$. 


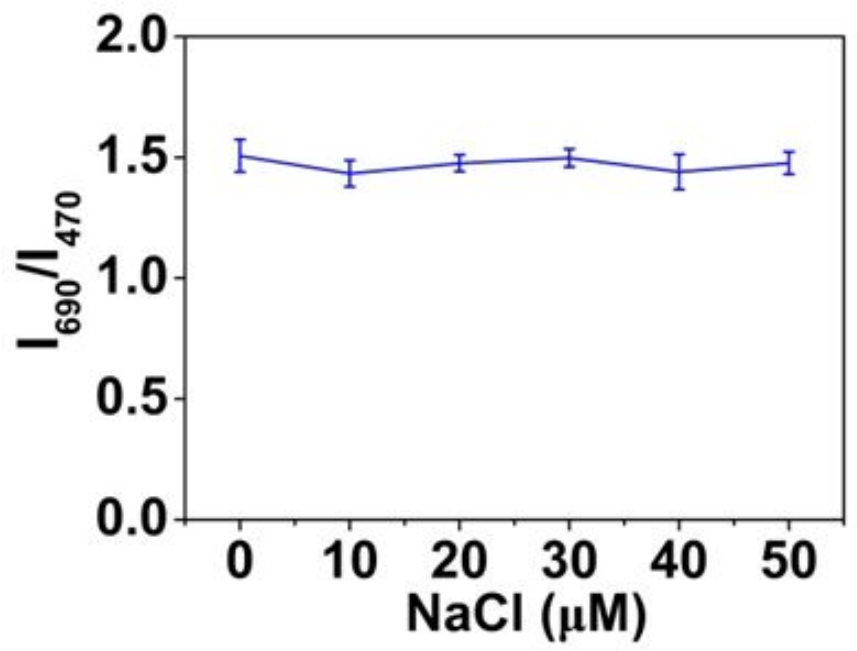

Figure S6. The effect of ionic strength on the fluorescence intensity ratio of the CDs + CdTe QDs hybrid solution.
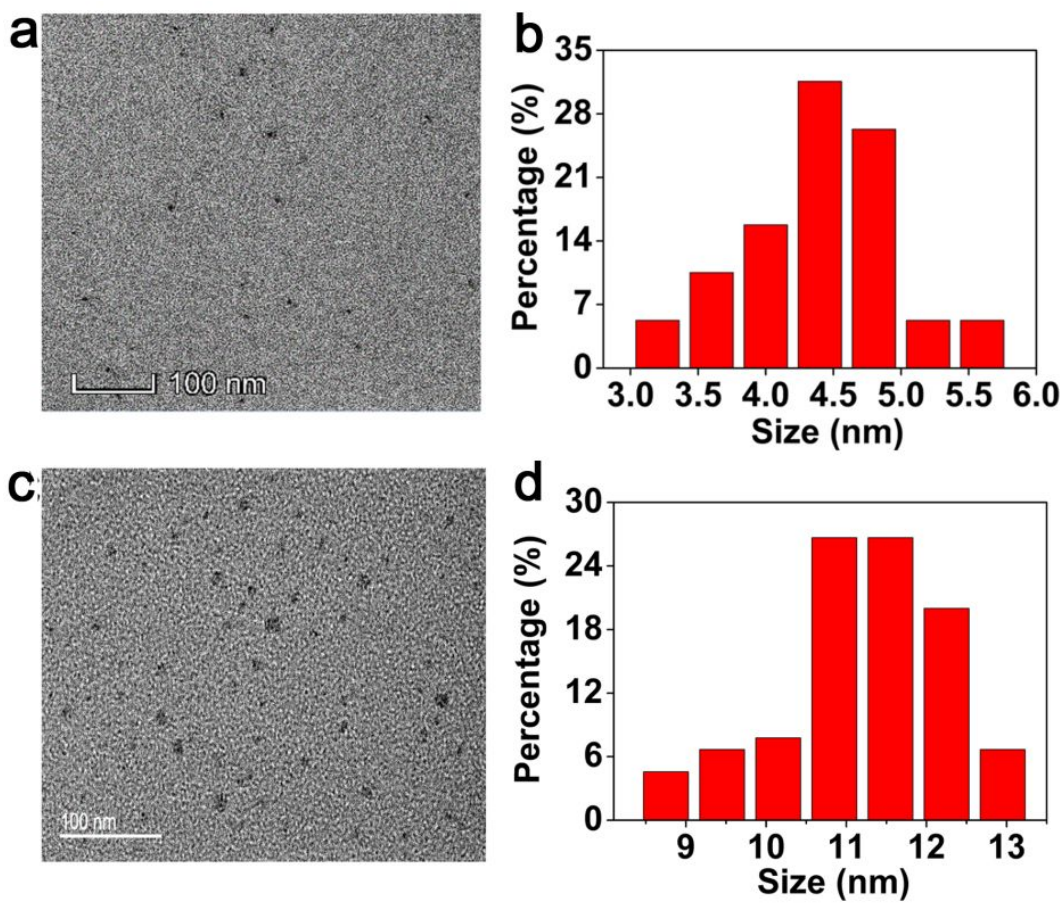

Figure S7. TEM images and particle size distribution histograms of CDs+CdTe QDs hybrid in the (a, and b) absence and (c and d) presence of spermine. 